\title{
Optical sensing by exposed core fiber using self-written waveguide
}

\begin{abstract}
Recently, the importance of the optical fiber is increasing particularly in sensing application. Thus, this paper proposes optical sensing by exposed core fiber technology using SelfWritten Waveguide (SWW) method. SWW method is fabrication of an optical channel waveguide using a UV-curable resin. As a UV light irradiated, A SWW with length of 800 micrometers is aligned between two fibers from the end of the multi-mode optical fiber. A several testing materials with different refractive index are being tested on the SWW. The output optical power is decreasing as the refractive indexes of testing materials are increasing. From these results, this makes the SWW technology is a reliable exposed core technology for sensing application.
\end{abstract}

Keyword: Sensing application; UV-curable resin; Self-Written Waveguide (SWW); Exposed core; Ray-tracing simulation 